\title{
Optimal CDMA Signature Sequences, Inverse Eigenvalue Problems and Alternating Minimization
}

\author{
Joel A. Tropp ${ }^{1}$ \\ Inst. for Comp. Engr. and Sci. \\ Univ. of Texas at Austin (C0200) \\ Austin, TX 78712, USA \\ e-mail: jtropp@ices.utexas.edu
}

\author{
Robert W. Heath, Jr. \\ Dept. of Elect. and Comp. Engr. \\ Univ. of Texas at Austin (C0803) \\ Austin, TX 78712, USA \\ e-mail: rheath@ece.utexas.edu
}

\author{
Thomas Strohmer \\ Dept. of Mathematics \\ Univ. of California at Davis \\ Davis, CA 95616, USA \\ e-mail: strohmer@math.ucdavis.edu
}

\section{INTRODUCTION AND BACKGROUND}

Recently, there has been an explosion of interest in optimal signature sequences for the synchronous code-division multiple-access (S-CDMA) channel in the presence of white noise. Proposed algorithms (e.g. [1]) exploit the relationship between minimizing the total squared correlation and maximizing the capacity of the channel [2]. In this abstract, we show how matrix-theoretic ideas can be mustered to solve these problems. It turns out that many types of sequence design fall into the category of structured inverse eigenvalue problems, which questions have been studied extensively [3]. We shall outline one technique for building optimal signatures in a particularly simple setting, with the understanding that our methods can be extended to more elaborate problems.

Specifically; we shall construct signature sequences that maximize the sum capacity of the S-CDMA channel with white noise and uniform received powers. Let $\left\{s_{n}\right\}_{n=1}^{N} \subset \mathbb{C}^{d}$ be unit-norm signatures, and form these column vectors into a $d \times N$ matrix $S$. A signature matrix achieves the channel capacity if and only if $d S S^{*}=N I_{d}$ [2]. Therefore, we must build $S$ whose $d$ singular values equal $\sqrt{N / d}$ and whose $N$ column norms equal one. Such matrices are known as WelchBound-Equality sequences or unit-norm tight frames.

\section{The Algorithm And Its Behavior}

One method for constructing matrices which satisfy several properties is to enforce those properties alternately in hope that the sequence will converge to a distinguished matrix. Let $\mathscr{S}$ be the collection of $d \times N$ full-rank matrices with unit columns, and let $\mathscr{Y}$ be the collection of $d \times N$ matrices with orthonormal rows and non-zero columns. Then we may define two operators $P: \mathscr{S} \longrightarrow \mathscr{Y}$ and $Q: \mathscr{Y} \longrightarrow \mathscr{S}$ by $P(S) \stackrel{\text { def }}{=}$ $\left(S S^{*}\right)^{-1 / 2} S$ and $Q(Y) \stackrel{\text { def }}{=} Y C^{-1}$, where $C$ is a diagonal matrix that lists the column norms of $Y$.

Algorithm 1 Let $S_{0} \in \mathscr{S}$. For each $j$, define $Y_{j} \stackrel{\text { def }}{=} P\left(S_{j}\right)$ and $S_{j+1} \stackrel{\text { dof }}{=} Q\left(Y_{j}\right)$. Repeat until the iterates have converged within a numerical tolerance.

It can be shown that each operator minimizes the Frobenius distance between its argument and the other constraint set, so this algorithm provides an example of alternating minimization. After verifying some technical conditions, we may appeal to a theorem of Meyer [4] to prove convergence. We conclude that the sequence of iterates is asymptotically regular, i.e. $\left\|S_{j+1}-S_{j}\right\|_{\mathrm{F}} \longrightarrow 0$, which implies that it either converges in norm or that it has a continuum of accumulation points. Every accumulation point is a fixed point of the algorithm, and these are easily characterized.

\footnotetext{
${ }^{1}$ Supported in part by NSF DMS-Grant 0208568.
}

Theorem 1 The fixed points of Algorithm 1 are precisely those matrices $S$ whose columns are all eigenvectors of $S S^{*}$. Equivalently, each stationary point is a union of mutually orthogonal Welch-Bound-Equality sequences. Moreover, the only stable fixed points are global maxima of the sum capacity.

We recommend initializing the algorithm with a random signature set since they converge almost surely to WBEs as the number of signatures increases. Experiments indicate that our algorithm converges much more rapidly than the method of Ulukus and Yates [1] for problems of realistic size. Nevertheless, the local convergence of an alternating minimization is linear at best. In the neighborhood of a stationary point, it would probably be more efficient to use a different type of iteration such as a projected gradient method.

\section{EXTENSIONS AND FURTHER WORK}

The intuition underlying our procedure suggests other avenues of research. First, we have shown that an obvious modification of Algorithm 1 can construct optimal signature sequences when the received powers of the users are not uniform. We have also developed methods for constructing signature sequences with additional properties. For example, by alternating between a spectral constraint and a unimodular constraint on the sequence components, we have succeeded in building unimodular signature sequences. We are also studying related techniques for more difficult sequence design problems such as the construction of Maximum Welch-Bound-Equality sequences. Finally, it is known that optimal sequences for the S-CDMA channel with colored noise also satisfy certain spectral properties [2]. It should be possible to develop alternating algorithms to construct sequences optimal for colored noise. In [5], we discuss some of these ideas at more length.

\section{ACKNOWLEDGMENTS}

Many thanks to Dr. I. S. Dhillon for helpful conversations.

\section{REFERENCES}

[1] S. Ulukus and R. D. Yates. "Iterative construction of optimal signature sequences in synchronous CDMA systems," IEEE Trans. IT, 47(5), 1989-1998, Jul. 2001.

[2] P. Viswanath and V. Anantharam. "Optimal sequences for CDMA under colored noise," IEEE Trans. IT, 48(6), 12951318, Jun. 2002.

[3] M. T. Chu and G. H. Golub. "Structured inverse eigenvalue problems," Acta Numerica, 11, 1-71, Jan. 2002.

[4] R. R. Meyer. "Sufficient conditions for the convergence of monotonic mathematical programming algorithms," J. Comp. Syst. Sci., 12, 108-121, 1976.

[5] J. A. Tropp, I. S. Dhillon, R. W. Heath Jr. and T. Strohmer. "Optimal CDMA signature sequences and inverse eigenvalue problems." In preparation, 2003. 